\title{
The Schur Multiplicative and Harmonic Convexities for Three Classes of Symmetric Functions
}

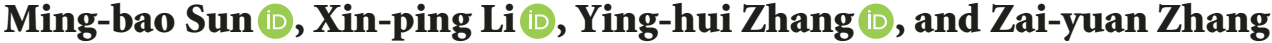 \\ School of Mathematics, Hunan Institute of Science and Technology, Yueyang, Hunan 414006, China \\ Correspondence should be addressed to Xin-ping Li; aslixp@163.com
}

Received 20 October 2018; Accepted 28 November 2018; Published 13 December 2018

Academic Editor: Alberto Fiorenza

Copyright (C) 2018 Ming-bao Sun et al. This is an open access article distributed under the Creative Commons Attribution License, which permits unrestricted use, distribution, and reproduction in any medium, provided the original work is properly cited.

We investigate the Schur harmonic convexity for two classes of symmetric functions and the Schur multiplicative convexity for a class of symmetric functions by using a new method and generalizing previous result. As applications, we establish some inequalities by use of the theory of majorization, in particular, and we give some new geometric inequalities in the $n$-dimensional space.

\section{Introduction}

Throughout this paper, we denote by $\mathbb{R}^{n}$ the $n$-dimensional Euclidean space, $\mathbb{R}=\mathbb{R}^{1}=(-\infty,+\infty)$ and $\mathbb{R}_{+}^{n}=\{x=$ $\left.\left(x_{1}, x_{2}, \ldots, x_{n}\right) \in \mathbb{R}^{n}: x_{i}>0, i=1,2, \ldots, n\right\}$. For $x=$ $\left(x_{1}, x_{2}, \ldots, x_{n}\right), y=\left(y_{1}, y_{2}, \ldots, y_{n}\right) \in \mathbb{R}^{n}$, and $\alpha \in \mathbb{R}$, we denote by

$$
\begin{aligned}
x+y & =\left(x_{1}+y_{1}, x_{2}+y_{2}, \ldots, x_{n}+y_{n}\right), \\
x y & =\left(x_{1} y_{1}, x_{2} y_{2}, \ldots, x_{n} y_{n}\right), \\
\alpha x & =\left(\alpha x_{1}, \alpha x_{2}, \ldots, \alpha x_{n}\right), \\
\alpha+x & =\left(\alpha+x_{1}, \alpha+x_{2}, \ldots, \alpha+x_{n}\right), \\
\frac{1}{x} & =\left(\frac{1}{x_{1}}, \frac{1}{x_{2}}, \ldots, \frac{1}{x_{n}}\right) .
\end{aligned}
$$

Furthermore, for $x=\left(x_{1}, x_{2}, \ldots, x_{n}\right) \in \mathbb{R}_{+}^{n}$, we denote by

$$
\begin{aligned}
\log x & =\left(\log x_{1}, \log x_{2}, \ldots, \log x_{n}\right), \\
x^{\alpha} & =\left(x_{1}^{\alpha}, x_{2}^{\alpha}, \ldots, x_{n}^{\alpha}\right) .
\end{aligned}
$$

Recently, the Schur convexity, Schur multiplicative convexity, and Schur harmonic convexity for some special functions and their applications have been investigated by many authors; see, for instance, [1-12] and the references therein.
For $x=\left(x_{1}, \ldots, x_{n}\right) \in[0,1)^{n}=\left\{x=\left(x_{1}, x_{2}, \ldots, x_{n}\right) \in\right.$ $\left.\mathbb{R}^{n}: 0 \leq x_{i}<1, i=1,2, \ldots, n\right\}$ and $r \in\{1,2, \ldots, n\}$, the symmetric function $F_{n}(x, r)$ was defined by Guan [13] as

$$
\begin{aligned}
F_{n}(x, r) & =F_{n}\left(x_{1}, x_{2}, \ldots, x_{n} ; r\right) \\
& =\sum_{1 \leq i_{1}<i_{2}<\cdots i_{r} \leq n} \prod_{j=1}^{r} \frac{x_{i_{j}}}{1-x_{i_{j}}},
\end{aligned}
$$

where $i_{1}, i_{2}, \ldots, i_{n}$ are positive integers. In [13], Guan discussed the Schur convexity and Schur multiplicative convexity for $F_{n}(x, r)$, established some interesting inequalities by use of the theory of majorization, and proposed an open problem as follows.

Open Problem A. If $n \geq 5$ and $r \in\{3, \ldots, n-2\}$, then the function $F_{n}(x, r)$ is Schur convex, or Schur concave.

Chu et al. [2] solved the Open Problem A and established the following Theorem A.

Theorem A. If $n \geq 2$ and $r \in\{2, \ldots, n\}$, then the function $F_{n}(x, r)$ is Schur concave in $[0,(r-1) / 2(n-1)]^{n}$ and Schur convex in $[(r-1) / 2(n-1), 1)^{n}$.

Xia and Chu [14] defined the following symmetric function:

$$
\begin{aligned}
G_{n}(x, r) & =G_{n}\left(x_{1}, x_{2}, \ldots, x_{n} ; r\right) \\
& =\sum_{1 \leq i_{1}<i_{2}<\cdots i_{r} \leq n} \prod_{j=1}^{r} \frac{1-x_{i_{j}}}{x_{i_{j}}},
\end{aligned}
$$


where $x=\left(x_{1}, \ldots, x_{n}\right) \in(0,1]^{n}, r \in\{1,2, \ldots, n\}$, and $i_{1}, i_{2}, \ldots, i_{n}$ are positive integers. In [14], they discussed the Schur convexity and Schur multiplicative convexity for $G_{n}(x, r)$ and established some inequalities.

For $x=\left(x_{1}, \ldots, x_{n}\right) \in \mathbb{R}_{+}^{n}$ and $r \in\{1,2, \ldots, n\}$, the symmetric function $M_{n}(x, r)$ was defined by Xia et al. [15] as

$$
\begin{aligned}
M_{n}(x, r) & =M_{n}\left(x_{1}, x_{2}, \ldots, x_{n} ; r\right) \\
& =\sum_{1 \leq i_{1}<i_{2}<\cdots i_{r} \leq n} \prod_{j=1}^{r} \frac{x_{i_{j}}}{1+x_{i_{j}}},
\end{aligned}
$$

where $i_{1}, i_{2}, \ldots, i_{n}$ are positive integers. In [15], Xia et al. proved that $M_{n}(x, r)$ is Schur concave and Schur harmonic convex in $\mathbb{R}_{+}^{n}$ (see [15, Theorems 3.1-3.2]) and Schur multiplicatively concave in $[1,+\infty)^{n}$, while $M_{n}(x, 1)$ is Schur multiplicatively convex in $(0,1]^{n}$, and $M_{n}(x, n)$ is Schur multiplicatively concave in $[1,+\infty)^{n}$ (see $[15$, Theorem 3.3]).

It is natural to ask whether the symmetric functions $F_{n}(x, r)$ and $G_{n}(x, r)$ are Schur harmonic convex, or Schur harmonic concave in $\mathbb{R}_{+}^{n}$, while the function $M_{n}(x, r)$ is Schur multiplicatively convex or Schur multiplicatively concave for $x \in(0,1)^{n}$ and $r \in\{2, \ldots, n-1\}$, which have not been studied in existing literatures.

The main purpose of this paper is to discuss the Schur harmonic convexity of $F_{n}(x, r)$ and $G_{n}(x, r)$ in $\mathbb{R}_{+}^{n}$ and solve the problem of the Schur multiplicative convexity or concavity of $M_{n}(x, r)$ in $(0,1)^{n}(r=2, \ldots, n-1)$ by using a new method, the main difficulty in proving Theorem 3 is to apply some techniques of differential calculus and combinatorics, in particular, and construct one complicate equality (29) in proof of Theorem 3. As applications, we establish some inequalities by use of the theory of majorization, in particular, we give some new geometric inequalities in the $n$ dimensional space.

Our main results are the following three theorems.

Theorem 1. For $x \in(0,1)^{n} \cup(1,+\infty)^{n}, n \geq 2$ and $r \in$ $\{1,2, \ldots, n\}$.

(1) The function $F_{n}(x, r)$ is Schur harmonic convex in

(2) If $r$ is even integer (or odd integer, respectively), then $F_{n}(x, r)$ is Schur harmonic convex (or concave, respectively) in $(1,+\infty)^{n}$.

Theorem 2. For $x \in(0,+\infty)^{n}, n \geq 2$ and $r \in\{1,2, \ldots, n\}$. $(0,1]^{n}$

(1) The function $G_{n}(x, r)$ is Schur harmonic concave in

(2) if $r$ is even integer (or odd integer, respectively), then $G_{n}(x, r)$ is Schur harmonic concave (or convex, respectively) in $(1,+\infty)^{n}$.

Theorem 3. For $x \in(0,+\infty)^{n}, n \geq 3$, and $r \in\{2, \ldots, n-1\}$, $M_{n}(x, r)$ is Schur multiplicatively convex in $(0,(n-r) /(n-1)]^{n}$ and Schur multiplicatively concave in $((n-r) /(n-1),+\infty)^{n}$.

Remark 4. From the proof of Theorem 3.3 in [15], it is easy to see that Theorem 3 is valid for $r=1$ and $r=n$, and Theorems 3 is a generalization of the Theorem 3.3 in [15], which is the main result in [15], thus, by using a new method we generalize and solve the problem of the Schur multiplicative convexity or concavity of $M_{n}(x, r)$ in $(0,1)^{n}(r=2, \ldots, n-1)$ from [15].

This paper, except for the introduction, is divided into three sections. In Section 2, we recall some definitions and lemmas. By using the results of Section 2, we give the proof of the main results in Section 3. Finally, some applications are given by use of the theory of majorization.

\section{Definitions and Lemmas}

For convenience, we recall some definitions.

Definition 5. Let $\Omega \subseteq \mathbb{R}^{n}$ be a set; a real-valued function $f$ on $\Omega$ is said to be Schur convex if

$$
f\left(x_{1}, x_{2}, \ldots, x_{n}\right) \leq f\left(y_{1}, y_{2}, \ldots, y_{n}\right)
$$

for each pair of $n$-tuples $x=\left(x_{1}, x_{2}, \ldots, x_{n}\right)$ and $y=$ $\left(y_{1}, y_{2}, \ldots, y_{n}\right)$ in $\Omega$, such that $x \prec y$, that is

$$
\sum_{i=1}^{k} x_{[i]} \leq \sum_{i=1}^{k} y_{[i]}, \quad k=1,2, \ldots, n-1
$$

and

$$
\sum_{i=1}^{n} x_{[i]}=\sum_{i=1}^{n} y_{[i]}
$$

where $x_{[i]}$ denotes the $i$ th largest component in $x . f$ is called Schur concave if $-f$ is Schur convex.

The above notion of Schur convexity was first introduced by Schur in 1923 and has many important applications in analytic inequalities [16-20], isoperimetric problem for polytopes [21], linear regression [22], combinatorial optimization [23], graphs and matrices [24], gamma and digamma functions [25], information-theoretic topics [26], stochastic orderings [27], and other related fields. The Definition 5 can be found in many references such as $[20,28]$. Following Schur, the following definition of the Schur multiplicative convex function was introduced in $[29,30]$.

Definition 6. Let $\Omega \subseteq \mathbb{R}_{+}^{n}$ be a set; a real-valued function $f$ on $\Omega$ is said to be Schur multiplicatively convex if

$$
f\left(x_{1}, x_{2}, \ldots, x_{n}\right) \leq f\left(y_{1}, y_{2}, \ldots, y_{n}\right)
$$

for each pair of $n$-tuples $x=\left(x_{1}, x_{2}, \ldots, x_{n}\right)$ and $y=$ $\left(y_{1}, y_{2}, \ldots, y_{n}\right)$ in $\Omega$, such that $\log x \prec \log y . f$ is called Schur multiplicatively concave if $1 / f$ is Schur multiplicatively convex.

Recently, Chu and Lv [31] introduced the notion of Schur harmonic convexity.

Definition 7. Let $\Omega \subseteq \mathbb{R}_{+}^{n}$ be a set; a real-valued function $f$ on $\Omega$ is said to be Schur harmonic convex function if

$$
f\left(x_{1}, x_{2}, \ldots, x_{n}\right) \leq f\left(y_{1}, y_{2}, \ldots, y_{n}\right),
$$


for each pair of $n$-tuples $x=\left(x_{1}, x_{2}, \ldots, x_{n}\right)$ and $y=$ $\left(y_{1}, y_{2}, \ldots, y_{n}\right)$ in $\Omega$, such that $1 / x<1 / y . f$ is called Schur harmonic concave if inequality (10) is reversed.

In order to establish our main result, we need two lemmas.

Lemma 8 (see $[29,30]$ ). Let $\Omega \subseteq \mathbb{R}_{+}^{n}$ be a symmetric multiplicatively convex set with nonempty interior in int $\Omega$ and $f: \Omega \longrightarrow \mathbb{R}_{+}^{1}$ be a continuous symmetry function on $\Omega$. If $f$ is differentiable in int $\Omega$, then $f$ is Schur multiplicatively convex on $\Omega$ if and only if

$$
\left(\log x_{1}-\log x_{2}\right)\left(x_{1} \frac{\partial f(x)}{\partial x_{1}}-x_{2} \frac{\partial f(x)}{\partial x_{2}}\right) \geq 0
$$

for all $x=\left(x_{1}, \ldots, x_{n}\right) \in$ int $\Omega$. And $f$ is Schur multiplicatively concave on $\Omega$ if and only if inequality (11) is reversed. Here $\Omega$ is a multiplicatively convex set which means that $x^{1 / 2} y^{1 / 2} \in \Omega$ for $x, y \in \Omega$.

Lemma 9 (see [31]). Let $\Omega \subseteq \mathbb{R}_{+}^{n}$ be a symmetric harmonic convex set with nonempty interior in int $\Omega$ and $f: \Omega \longrightarrow \mathbb{R}_{+}^{1}$ be a continuous symmetry function on $\Omega$. If $f$ is differentiable on int $\Omega$, then $f$ is Schur harmonic convex on $\Omega$ if and only if

$$
\left(x_{1}-x_{2}\right)\left(x_{1}^{2} \frac{\partial f(x)}{\partial x_{1}}-x_{2}^{2} \frac{\partial f(x)}{\partial x_{2}}\right) \geq 0
$$

for all $x=\left(x_{1}, \ldots, x_{n}\right) \in$ int $\Omega$. And $f$ is Schur harmonic concave in $\Omega$ if and only if inequality (12) is reversed. Here $\Omega$ is a harmonic convex set which means that $2 x y /(x+y) \in \Omega$ for $x, y \in \Omega$.

\section{Proofs of the Main Results}

Proof of Theorem 1. (1) According to Lemma 9, we only need to prove that

$$
\begin{aligned}
P_{n}(x, r) & =\left(x_{1}-x_{2}\right)\left(x_{1}^{2} \frac{\partial F_{n}(x, r)}{\partial x_{1}}-x_{2}^{2} \frac{\partial F_{n}(x, r)}{\partial x_{2}}\right) \\
& \geq 0
\end{aligned}
$$

for all $x \in(0,1)^{n}$ and $r \in\{1,2, \ldots, n\}$. The proof is divided into three cases.

Case 1. If $r=1$, then from (3) we have

$$
\begin{aligned}
F_{n}(x, 1) & =\sum_{i=1}^{n} \frac{x_{i}}{1-x_{i}}, \\
\frac{\partial F_{n}(x, 1)}{\partial x_{i}} & =\frac{1}{\left(1-x_{i}\right)^{2}} . \quad i=1,2
\end{aligned}
$$

By (15), we obtain

$$
\begin{aligned}
P_{n}(x, 1) & =\left(x_{1}-x_{2}\right)\left(x_{1}^{2} \frac{\partial F_{n}(x, 1)}{\partial x_{1}}-x_{2}^{2} \frac{\partial F_{n}(x, 1)}{\partial x_{2}}\right) \\
& =\frac{\left(x_{1}-x_{2}\right)^{2}\left[x_{1}\left(1-x_{2}\right)+x_{2}\left(1-x_{1}\right)\right]}{\left(1-x_{1}\right)^{2}\left(1-x_{2}\right)^{2}}
\end{aligned}
$$

Case 2. If $n \geq 2$ and $r=n$, then from (3) we obtain

$$
\begin{aligned}
F_{n}(x, n) & =\prod_{i=1}^{n} \frac{x_{i}}{1-x_{i}}, \\
\frac{\partial F_{n}(x, n)}{\partial x_{i}} & =\frac{F_{n}(x, n)}{x_{i}\left(1-x_{i}\right)} . \quad i=1,2
\end{aligned}
$$

By (18), we obtain

$$
\begin{aligned}
P_{n}(x, n) & =\left(x_{1}-x_{2}\right)\left(x_{1}^{2} \frac{\partial F_{n}(x, n)}{\partial x_{1}}-x_{2}^{2} \frac{\partial F_{n}(x, n)}{\partial x_{2}}\right) \\
& =\frac{\left(x_{1}-x_{2}\right)^{2} F_{n}(x, n)}{\left(1-x_{1}\right)\left(1-x_{2}\right)} \geq 0 .
\end{aligned}
$$

Case 3. If $n \geq 3$ and $2 \leq r \leq n-1$, noting that the $r$-th order elementary symmetric function $E_{n}(x, r)$ (see [32]) is defined as

$$
\begin{aligned}
E_{n}(x, r) & =E_{n}\left(x_{1}, x_{2}, \ldots, x_{n} ; r\right) \\
& =\sum_{1 \leq i_{1}<i_{2}<\cdots<i_{r} \leq n} \prod_{j=1}^{r} x_{i_{j}},
\end{aligned}
$$

where $x=\left(x_{1}, \ldots, x_{n}\right) \in \mathbb{R}_{+}^{n}, r \in\{1,2, \ldots, n\}$, and $i_{1}, i_{2}, \ldots, i_{n}$ are positive integers and $E_{n}(x, 0)=E_{n}\left(x_{1}, x_{2}, \ldots, x_{n} ; 0\right)=1$.

Let $u_{i}=x_{i} /\left(1-x_{i}\right), i=1, \ldots, n$, and then from (3), we have

$$
\begin{aligned}
F_{n}(x, r) & \\
= & \frac{x_{1} x_{2}}{\left(1-x_{1}\right)\left(1-x_{2}\right)} E_{n-2}\left(u_{3}, u_{4}, \ldots, u_{n} ; r-2\right) \\
& +\frac{x_{1}}{1-x_{1}} E_{n-2}\left(u_{3}, u_{4}, \ldots, u_{n} ; r-1\right) \\
& +E_{n-1}\left(u_{2}, \ldots, u_{n} ; r\right) \\
= & \frac{x_{1} x_{2}}{\left(1-x_{1}\right)\left(1-x_{2}\right)} E_{n-2}\left(u_{3}, u_{4}, \ldots, u_{n} ; r-2\right) \\
& +\frac{x_{2}}{1-x_{2}} E_{n-2}\left(u_{3}, u_{4}, \ldots, u_{n} ; r-1\right) \\
& +E_{n-1}\left(u_{1}, u_{3}, \ldots, u_{n} ; r\right),
\end{aligned}
$$

$$
\begin{aligned}
& \frac{\partial F_{n}(x, r)}{\partial x_{1}} \\
& =\frac{x_{2}}{\left(1-x_{1}\right)^{2}\left(1-x_{2}\right)} E_{n-2}\left(u_{3}, u_{4}, \ldots, u_{n} ; r-2\right) \\
& \quad+\frac{1}{\left(1-x_{1}\right)^{2}} E_{n-2}\left(u_{3}, u_{4}, \ldots, u_{n} ; r-1\right),
\end{aligned}
$$

$$
\begin{aligned}
& \frac{\partial F_{n}(x, r)}{\partial x_{2}} \\
& =\frac{x_{1}}{\left(1-x_{1}\right)\left(1-x_{2}\right)^{2}} E_{n-2}\left(u_{3}, u_{4}, \ldots, u_{n} ; r-2\right) \\
& \quad+\frac{1}{\left(1-x_{2}\right)^{2}} E_{n-2}\left(u_{3}, u_{4}, \ldots, u_{n} ; r-1\right),
\end{aligned}
$$


From (22) and (23), we obtain

$$
\begin{aligned}
& P_{n}(x, r)=\left(x_{1}-x_{2}\right)\left(x_{1}^{2} \frac{\partial F_{n}(x, r)}{\partial x_{1}}-x_{2}^{2} \frac{\partial F_{n}(x, r)}{\partial x_{2}}\right) \\
& =\frac{\left(x_{1}-x_{2}\right)^{2}}{\left.\left(1-x_{1}\right)^{2} 1-x_{2}\right)^{2}}\left\{x _ { 1 } x _ { 2 } E _ { n - 2 } \left(u_{3}, u_{4}, \ldots, u_{n} ; r\right.\right. \\
& \quad-2)+\left[x_{1}\left(1-x_{2}\right)+x_{2}\left(1-x_{1}\right)\right] \\
& \left.\cdot E_{n-2}\left(u_{3}, u_{4}, \ldots, u_{n} ; r-1\right)\right\} \geq 0,
\end{aligned}
$$

From Cases 1-3 we obtain that (13) holds for all $x \in(0,1)^{n}$ and $r \in\{1,2, \ldots, n\}$; therefore, $F_{n}(x, r)$ is schur harmonic convex in $(0,1)^{n}$.

(2) Here we give only the proof in the case of $r$ being even integer, since the proof in the case of $r$ being odd integer is similar. According to Lemma 9, we only need to prove that (13) holds for all $x \in(1,+\infty)^{n}$. From the proofs of Cases 23 in (1), it is easy to see that $P_{n}(x, r) \geq 0$ for $x \in(1,+\infty)^{n}$; thus we derive that (13) holds for all $x \in(1,+\infty)^{n}$ in the case of $r$ being even integer; therefore, $F_{n}(x, r)$ is Schur harmonic convex in $(1,+\infty)^{n}$.

The proof of Theorem 1 is completed.

Similar to the proof of Theorem 1, we can use Lemma 9 and (20) to prove Theorem 2; therefore we omit the details of the proof. Next we give the proof of Theorem 3 .

Proof of Theorem 3. According to Lemma 8, we only need to discuss the nonnegativity and nonpositivity of $Q_{n}(x, r)=$ $\left(\log x_{1}-\log x_{2}\right)\left(x_{1}\left(\partial M_{n}(x, r) / \partial x_{1}\right)-x_{2}\left(\partial M_{n}(x, r) / \partial x_{2}\right)\right)$ for all $x \in(0,+\infty)^{n}$ and $r \in\{2, \ldots, n-1\}$.

Let $v_{i}=x_{i} /\left(1+x_{i}\right), i=1, \ldots, n$; then from (5) and (20) we have

$$
\begin{aligned}
M_{n}(x, r) & \\
= & \frac{x_{1} x_{2}}{\left(1+x_{1}\right)\left(1+x_{2}\right)} E_{n-2}\left(v_{3}, v_{4}, \ldots, v_{n} ; r-2\right) \\
& +\frac{x_{1}}{1+x_{1}} E_{n-2}\left(v_{3}, v_{4}, \ldots, v_{n} ; r-1\right) \\
& +E_{n-1}\left(v_{2}, \ldots, v_{n} ; r\right) \\
= & \frac{x_{1} x_{2}}{\left(1+x_{1}\right)\left(1+x_{2}\right)} E_{n-2}\left(v_{3}, v_{4}, \ldots, v_{n} ; r-2\right) \\
& +\frac{x_{2}}{1+x_{2}} E_{n-2}\left(v_{3}, v_{4}, \ldots, v_{n} ; r-1\right) \\
& +E_{n-1}\left(v_{1}, v_{3}, \ldots, v_{n} ; r\right), \\
\left.\frac{\partial E_{n}(}{\partial x_{1}} r\right) & \\
= & \frac{1+x_{2}}{x_{1} f\left(x_{1}\right) f\left(x_{2}\right)} E_{n-2}\left(v_{3}, v_{4}, \ldots, v_{n} ; r-2\right) \\
& +\frac{1}{x_{1} f\left(x_{1}\right)} E_{n-2}\left(v_{3}, v_{4}, \ldots, v_{n} ; r-1\right),
\end{aligned}
$$

$$
\begin{aligned}
& \frac{\partial E_{n}(x, r)}{\partial x_{2}} \\
& =\frac{1+x_{1}}{x_{2} f\left(x_{1}\right) f\left(x_{2}\right)} E_{n-2}\left(v_{3}, v_{4}, \ldots, v_{n} ; r-2\right) \\
& \quad+\frac{1}{x_{2} f\left(x_{2}\right)} E_{n-2}\left(v_{3}, v_{4}, \ldots, v_{n} ; r-1\right),
\end{aligned}
$$

where $f(x)=(1+x)^{2} / x$. From (26) and (27) we obtain

$$
\begin{aligned}
& Q_{n}(x, r) \\
& =\left(\log x_{1}-\log x_{2}\right)\left(x_{1} \frac{\partial M_{n}(x, r)}{\partial x_{1}}-x_{2} \frac{\partial M_{n}(x, r)}{\partial x_{2}}\right) \\
& =-\frac{\log x_{1}-\log x_{2}}{f\left(x_{1}\right) f\left(x_{2}\right)}\left[g\left(x_{1}, r\right)-g\left(x_{2}, r\right)\right],
\end{aligned}
$$

where $g(x, r)=x E_{n-2}\left(v_{3}, v_{4}, \ldots, v_{n} ; r-2\right)+f(x) E_{n-2}\left(v_{3}, v_{4}\right.$, $\left.\ldots, v_{n} ; r-1\right)$. Note that

$$
\begin{aligned}
& E_{n-2}\left(v_{3}, v_{4}, \ldots, v_{n} ; r-1\right)=\frac{1}{r-1} \\
& \cdot \sum_{3 \leq i_{3}<\cdots<i_{r} \leq n} \prod_{j=3}^{r} \frac{x_{i_{j}}}{1+x_{i_{j}}}\left(\sum_{\substack{i_{k} \in\{3, \ldots, n\} \\
i_{k} \neq i_{j}(j=3, \ldots, n)}} \frac{x_{i_{k}}}{1+x_{i_{k}}}\right) \text {, } \\
& f^{\prime}(x)=\frac{x^{2}-1}{x^{2}} \\
& f^{\prime \prime}(x)=2 x^{-3}, \\
& \frac{\partial g(x, r)}{\partial x}=E_{n-2}\left(v_{3}, v_{4}, \ldots, v_{n} ; r-2\right)+f^{\prime}(x) \\
& \cdot E_{n-2}\left(v_{3}, v_{4}, \ldots, v_{n} ; r-1\right) \\
& =\sum_{3 \leq i_{3}<\cdots<i_{r} \leq n} \prod_{j=3}^{r} \frac{x_{i_{j}}}{1+x_{i_{j}}}(1 \\
& \left.+\frac{1}{r-1} f^{\prime}(x) \sum_{\substack{i_{k} \in\{3, \ldots, n\} \\
i_{k} \neq i_{j}(j=3, \ldots, n)}} \frac{x_{i_{k}}}{1+x_{i_{k}}}\right) \text {, }
\end{aligned}
$$

and

$$
\frac{\partial^{2} g(x, r)}{\partial x^{2}}=f^{\prime \prime}(x) E_{n-2}\left(v_{3}, v_{4}, \ldots, v_{n} ; r-1\right) \text {. }
$$

Let $A=(n-r) /(n-1)$; then $0<A<1$, from (30) we have

$$
S=1+\frac{n-r}{r-1} f^{\prime}(A)\left(\frac{A}{1+A}\right)=0
$$

From (31), we have $f^{\prime \prime}(x)>0$ for $x \in(0,+\infty)$; hence by (33), if $x_{i} \in(0,+\infty)(i=3,4, \ldots, n)$, then $\partial^{2} g(x, r) / \partial x^{2}>$ 
0 for $x \in(0,+\infty)$; therefore, $\partial g(x, r) / \partial x$ is increasing on $(0,+\infty)$ with $x$; further from (32) and (34), we obtain for $x, x_{i} \in(0, A)(i=3,4, \ldots, n)$

$$
\begin{gathered}
\frac{\partial g(x, r)}{\partial x}<\frac{\partial g(A, r)}{\partial x}=\sum_{3 \leq i_{3}<\cdots<i_{r} \leq n} \prod_{j=3}^{r} \frac{x_{i_{j}}}{1+x_{i_{j}}}{ }^{\prime} 1 \\
\left.+\frac{1}{r-1} f^{\prime}(A) \sum_{\substack{i_{k} \in\{3, \ldots, n\} \\
i_{k} \neq i_{j}(j=3, \ldots, n)}} \frac{x_{i_{k}}}{1+x_{i_{k}}}\right) \leq S M_{n-2}(A, \\
A, \ldots, A ; r-2)=0
\end{gathered}
$$

and have for $x, x_{i} \in(A,+\infty)(\mathrm{i}=3,4, \ldots, \mathrm{n})$

$$
\begin{aligned}
& \frac{\partial g(x, r)}{\partial x}>\frac{\partial g(A, r)}{\partial x}=\sum_{3 \leq i_{3}<\cdots<i_{r} \leq n} \prod_{j=3}^{r} \frac{x_{i_{j}}}{1+x_{i_{j}}}(1 \\
& \left.+\frac{1}{r-1} f^{\prime}(A) \sum_{\substack{i_{k} \in\{3, \ldots, n\} \\
i_{k} \neq i_{j}(j=3, \ldots, n)}} \frac{x_{i_{k}}}{1+x_{i_{k}}}\right) \geq S M_{n-2}(A, \\
& A, \ldots, A ; r-2)=0 .
\end{aligned}
$$

Hence by (35) and (36), respectively, if $x_{i} \in(0, A)(i=$ $3,4, \ldots, n)$, then $g(x, r)$ is decreasing on $(0, A)$ with $x$, and if $x_{i} \in(A,+\infty)(i=3,4, \ldots, n)$, then $g(x, r)$ is increasing on $(A,+\infty)$ with $x$; further from (28) we derive that $Q_{n}(x, r) \geq 0$ for $x \in(0, A)^{n}$, and $Q_{n}(x, r) \leq 0$ for $x \in(A,+\infty)^{n}$. Therefore, from Lemma $8, M_{n}(x, r)$ is Schur multiplicatively convex in $(0,(n-r) /(n-1)]^{n}$ and Schur multiplicatively concave in $((n-r) /(n-1),+\infty)^{n}$.

The proof of Theorem 3 is completed.

Remark 10. It is easy to see that the key point of our proof of Theorem 3 is to construct one monotone function $g(x, r)$ and prove that $g(x, r)$ is decreasing on $(0, A)$, and increasing on $(A,+\infty)$ with $x$, which is different from the proof of Theorem 3.3 in [15].

\section{Applications}

In this section, we establish some inequalities by use of Theorems 1-3 and the theory of majorization.

Theorem 11. Let $n \geq 2, x=\left(x_{1}, x_{2}, \ldots, x_{n}\right), A_{n}(x)=$ $(1 / n) \sum_{i=1}^{n} x_{i}$, and $r \in\{1,2, \ldots, n\}$.

(1) Suppose that $x \in(0,1)^{n}$. If $r$ is even integer, then

$$
\begin{gathered}
\sum_{1 \leq i_{1}<i_{2}<\cdots<i_{r} \leq n} \prod_{j=1}^{r} \frac{1}{x_{i_{j}}-1} \geq \frac{n !}{r !(n-r) !}\left[\frac{1}{A_{n}(x-1)}\right]^{r}, \\
\sum_{1 \leq i_{1}<i_{2}<\cdots<i_{r} \leq n} \prod_{j=1}^{r}\left(x_{i_{j}}-1\right) \leq \frac{n !}{r !(n-r) !}\left[A_{n}(x-1)\right]^{r},
\end{gathered}
$$

while if $r$ is odd integer, then the inequalities (37) and (38) are reversed

(2) If $x \in(1,+\infty)^{n}$, the inequalities (37) and (38) hold.

Proof. It is easy to see that

$$
\left(A_{n}(x), A_{n}(x), \ldots, A_{n}(x)\right) \prec\left(x_{1}, x_{2}, \ldots, x_{n}\right) .
$$

From Theorems 1 and 2, respectively, and applying (39), we get that Theorem 11 holds.

Theorem 12. Let $n \geq 2, x=\left(x_{1}, x_{2}, \ldots, x_{n}\right), H_{n}(x)=$ $n / \sum_{i=1}^{n}\left(1 / x_{i}\right)$, and $r \in\{1,2, \ldots, n\}$.

(1) If $x \in(0,1)^{n}$, then

$$
\begin{gathered}
\sum_{1 \leq i_{1}<i_{2}<\cdots<i_{r} \leq n} \prod_{j=1}^{r} \frac{x_{i_{j}}}{1-x_{i_{j}}} \geq \frac{n !}{(n-r) ! r !}\left[\frac{H_{n}(x)}{H_{n}(x)-1}\right]^{r}, \\
\sum_{1 \leq i_{1}<i_{2}<\cdots<i_{r} \leq n} \prod_{j=1}^{r} \frac{1-x_{i_{j}}}{x_{i_{j}}} \leq \frac{n !}{(n-r) ! r !}\left[\frac{1-H_{n}(x)}{H_{n}(x)}\right]^{r} ;
\end{gathered}
$$

(2) Suppose that $x \in(1,+\infty)^{n}$. If $r$ is even integer, then the inequalities (40) and (41) hold, while if $r$ is odd integer, the inequalities (40) and (41) are reversed.

Proof. It is easy to see that

$$
\left(\frac{1}{H_{n}(x)}, \frac{1}{H_{n}(x)}, \ldots, \frac{1}{H_{n}(x)}\right) \prec\left(\frac{1}{x_{1}}, \frac{1}{x_{2}}, \ldots, \frac{1}{x_{n}}\right) \text {. }
$$

From Theorems 1 and 2, respectively, and applying (42), we obtain that Theorem 12 holds.

Theorem 13. Let $n \geq 2, x=\left(x_{1}, x_{2}, \ldots, x_{n}\right), G_{n}(x)=$ $\left(\prod_{i=1}^{n} x_{i}\right)^{1 / n}$, and $r \in\{1,2, \ldots, n\}$. If $x \in(0,(n-r) /(n-1)]^{n}$, then

$$
\sum_{1 \leq i_{1}<i_{2}<\cdots i_{r} \leq n} \prod_{j=1}^{r} \frac{x_{i_{j}}}{1+x_{i_{j}}} \geq \frac{n !}{(n-r) ! r !}\left[\frac{G_{n}(x)}{1+G_{n}(x)}\right]^{r},
$$

if $x \in[(n-r) /(n-1),+\infty)^{n}$, the inequality (43) is reversed.

Proof. It is easy to see that

$$
\begin{aligned}
& \log \left(G_{n}(x), G_{n}(x), \ldots, G_{n}(x)\right) \\
& \quad \prec \log \left(x_{1}, x_{2}, \ldots, x_{n}\right) .
\end{aligned}
$$

From Theorems 3 and Remark 4, and applying (44), we obtain that Theorem 13 holds.

Remark 14. It is easy to see that Theorem 13 is a generalization of the Theorem 4.5 in [15].

Theorem 15. Let $\mathscr{A}=A_{1} A_{2} \cdots A_{n+1}$ be an $n$-dimensional simples in $R^{n}$ and $P$ be an arbitrary point in the interior of $\mathscr{A}$. If $B_{i}$ is the intersection point of straight $A_{i} P$ and hyperplane 
$\sum_{i}=A_{1} A_{2} \cdots A_{i-1} A_{i+1} \cdots A_{n+1}(i=1,2, \ldots, n+1)$. Then for $r \in\{1,2, \ldots, n+1\}$

$$
\begin{aligned}
\sum_{1 \leq i_{1}<i_{2}<\cdots<i_{r} \leq n+1} & \prod_{j=1}^{r} \frac{A_{i_{j}} B_{i_{j}}}{P A_{i_{j}}} \geq \frac{(n+1) !}{(n+1-r) ! r !}\left(\frac{n+1}{n}\right)^{r}, \\
\sum_{1 \leq i_{1}<i_{2}<\cdots<i_{r} \leq n+1} \prod_{j=1}^{r} \frac{P A_{i_{j}}}{A_{i_{j}} B_{i_{j}}} & \leq \frac{(n+1) !}{(n+1-r) ! r !}\left(\frac{n}{n+1}\right)^{r}, \\
\sum_{1 \leq i_{1}<i_{2}<\cdots<i_{r} \leq n+1} \prod_{j=1}^{r} \frac{A_{i_{j}} B_{i_{j}}}{P B_{i_{j}}} & \geq \frac{(n+1) !}{(n+1-r) ! r !}(n+1)^{r}, \\
\sum_{1 \leq i_{1}<i_{2}<\cdots<i_{r} \leq n+1} \prod_{j=1}^{r} \frac{P B_{i_{j}}}{A_{i_{j}} B_{i_{j}}} & \leq \frac{(n+1) !}{(n+1-r) ! r !}\left(\frac{1}{n+1}\right)^{r},
\end{aligned}
$$

Proof. It is easy to see that $\sum_{i=1}^{n+1}\left(P B_{i} / A_{i} B_{i}\right)=1$ and $P A_{i} / A_{i} B_{i}=1-P B_{i} / A_{i} B_{i}, i=1,2, \ldots, n+1$; these imply that

$$
\begin{aligned}
& \left(\frac{1}{n+1}, \frac{1}{n+1}, \ldots, \frac{1}{n+1}\right) \\
& \quad \prec\left(\frac{P B_{1}}{A_{1} B_{1}}, \frac{P B_{2}}{A_{2} B_{2}}, \ldots, \frac{P B_{n+1}}{A_{n+1} B_{n+1}}\right), \\
& \left(\frac{n}{n+1}, \frac{n}{n+1}, \ldots, \frac{n}{n+1}\right) \\
& \quad \prec\left(\frac{P A_{1}}{A_{1} B_{1}}, \frac{P A_{2}}{A_{2} B_{2}}, \ldots, \frac{P A_{n+1}}{A_{n+1} B_{n+1}}\right) .
\end{aligned}
$$

Therefore, (45)-(48) follow from Theorems 1(2) and 2(2), respectively, together with (49) and (50).

Remark 16. The inequalities (45)-(48) can be found in [33, p.473-480]; here we give a different proof of them.

Theorem 17. Let $\mathscr{A}=A_{1} A_{2} \cdots A_{n+1}$ be an $n$-dimensional simples in $R^{n}$ and $r$ be the inradius of $\mathscr{A}$. Let $h_{i}$ be the altitude of $A$ from the vertex $A_{i}$, i.e., the distance from $A_{i}$ to hyperplane $\sum_{i}=A_{1} A_{2} \cdots A_{i-1} A_{i+1} \cdots A_{n+1}(i=1,2, \ldots, n+1)$, and $r_{i}$ be the radius of $i$-th escribed hypersphere of $\mathscr{A}$. Then for $k \in\{1,2, \ldots, n+1\}$

$$
\begin{aligned}
\sum_{1 \leq i_{1}<i_{2}<\cdots<i_{k} \leq n+1} & \prod_{j=1}^{k} \frac{r_{i_{j}}}{r_{i_{j}}-r} \geq \frac{(n+1) !}{(n+1-k) ! k !}\left(\frac{n+1}{2}\right)^{k}, \\
\sum_{1 \leq i_{1}<i_{2}<\cdots<i_{k} \leq n+1} \prod_{j=1}^{k} \frac{r_{i_{j}}-r}{r_{i_{j}}} & \leq \frac{(n+1) !}{(n+1-k) ! k !}\left(\frac{2}{n+1}\right)^{k}, \\
\sum_{1 \leq i_{1}<i_{2}<\cdots<i_{k} \leq n+1} \prod_{j=1}^{k} \frac{h_{i_{j}}}{h_{i_{j}}-r} & \geq \frac{(n+1) !}{(n+1-k) ! k !}\left(\frac{n+1}{n}\right)^{k}, \\
\sum_{1 \leq i_{1}<i_{2}<\cdots<i_{k} \leq n+1} \prod_{j=1}^{k} \frac{h_{i_{j}}-r}{h_{i_{j}}} & \geq \frac{(n+1) !}{(n+1-k) ! k !}\left(\frac{n}{n+1}\right)^{k},
\end{aligned}
$$

Proof. Let $V$ denotes the volume of $\mathscr{A}, F_{i}(i=1,2, \ldots, n+$ $1)$ denote the $(n-1)$-dimensional volume of the side face of
$\mathscr{A}$ opposite to the vertex $A_{i}(\mathrm{i}=1,2, \ldots, \mathrm{n}+1)$. Let $F=\sum_{i=1}^{n+1} F_{i}$; noting the well-known fact (see [33, p.463])

$$
\begin{aligned}
r & =\frac{n V}{F}, \\
h_{i} & =\frac{n V}{F_{i}}, \\
r_{i} & =\frac{n V}{F-2 F_{i}},
\end{aligned}
$$

we obtain

$$
\begin{aligned}
& \frac{r}{r_{i}}=\frac{F-2 F_{i}}{F}, \\
& \frac{r}{h_{i}}=\frac{F_{i}}{F} .
\end{aligned}
$$

Thus we have

$$
\begin{aligned}
& \sum_{i=1}^{n+1} \frac{r}{r_{i}}=n-1, \\
& \sum_{i=1}^{n+1} \frac{r}{h_{i}}=1,
\end{aligned}
$$

and further we obtain

$$
\begin{aligned}
& \left(\frac{n-1}{n+1}, \frac{n-1}{n+1}, \ldots, \frac{n-1}{n+1}\right) \prec\left(\frac{r}{r_{1}}, \frac{r}{r_{2}}, \ldots, \frac{r}{r_{n+1}}\right), \\
& \left(\frac{1}{n+1}, \frac{1}{n+1}, \ldots, \frac{1}{n+1}\right) \prec\left(\frac{r}{h_{1}}, \frac{r}{h_{2}}, \ldots, \frac{r}{h_{n+1}}\right) .
\end{aligned}
$$

Therefore, (51)-(54) follow from Theorems 1(2) and 2(2), respectively, together with (58) and (59).

Remark 18. The inequality (51) can be found in the Proposition 4 from [19]; here we give a different proof of it. Mitrinović, Pečarić, and Volenec (see [33, p.463-473]) established a series of inequalities for $r, h_{i}$, and $r_{i}$; obviously, our inequalities (52)-(54) in Theorem 17 are different from theirs.

\section{Conclusions}

In this paper, we investigate the Schur harmonic convexity for two classes of symmetric functions defined by Guan (2007), Xia and Chu (2009), and the Schur multiplicative convexity for a class of symmetric functions defined by Xia et al. (2010) by using a new method and generalize the main results of Xia et al. (2010). As applications, we establish some inequalities by use of the theory of majorization; in particular, we give some new geometric inequalities in the $n$-dimensional space. It is easy to see that

(1) Our Theorem 13 is a generalization of the Theorem 4.5 in Xia et al. (2010).

(2) The inequalities (45)-(48) can be found in Mitrinović (1989); here we give a different proof of them.

(3) The inequality (51) can be found in the Proposition 4 from $\mathrm{Wu}$ (2005); here we give a different proof of it. 


\section{Data Availability}

No data of any type is used in this article.

\section{Conflicts of Interest}

The authors declare that there are no conflicts of interest regarding the publication of this paper.

\section{Acknowledgments}

This work was supported in part by Aid Program for Science and Technology Innovative Research Team in Higher Educational Institutions of Hunan Province, Nature Science Foundation of Hunan Province (Grant no. 12JJ3002), and National Nature Science Foundation of China (Grant no. 11271118).

\section{References}

[1] J. Sándor, "The Schur-convexity of STOlarsky and Gini means," Banach Journal of Mathematical Analysis, vol. 1, no. 2, pp. 212215, 2007.

[2] Y. Chu, W. Xia, and T. Zhao, "Schur convexity for a class of symmetric functions," Science China Mathematics, vol. 53, no. 2, pp. 465-474, 2010.

[3] Y.-M. Chu, G.-D. Wang, and X.-H. Zhang, "The Schur multiplicative and harmonic convexities of the complete symmetric function," Mathematische Nachrichten, vol. 284, no. 5-6, pp. 653-663, 2011.

[4] Y.-M. Chu, W.-F. Xia, and X.-H. Zhang, "The SCHur concavity, SCHur multiplicative and harmonic convexities of the second dual form of the Hamy symmetric function with applications," Journal of Multivariate Analysis, vol. 105, pp. 412-421, 2012.

[5] K. Guan, "The Hamy symmetric function and its generalization," Mathematical Inequalities \& Applications, vol. 9, no. 4, pp. 797-805, 2006

[6] K. Guan and R. Guan, "Some properties of a generalized Hamy symmetric function and its applications," Journal of Mathematical Analysis and Applications, vol. 376, no. 2, pp. 494505, 2011.

[7] K. M. Nagaraja, P. S. Reddy, and K. Sridevi, "Schur harmonic convexity of Gnan mean for two variables," Journal of International Mathematical Virtual Institute, vol. 3, pp. 61-80, 2013.

[8] M. Revyakov, "Schur-convexity of 2nd order, certain subclass of multivariate arrangement increasing functions with applications in statistics," Journal of Multivariate Analysis, vol. 116, pp. 25-34, 2013.

[9] H. N. Shi, Theory of Majorization and Analytic Inequalities, Harbin Institute of Technology Press, Harbin, China, 2012.

[10] H.-N. Shi and J. Zhang, "SCHur-convexity, SCHur geometric and SCHur harmonic convexities of dual form of a class symmetric functions," Journal of Mathematical Inequalities, vol. 8, no. 2, pp. 349-358, 2014.

[11] W. Xia and Y. Chu, "The Schur convexity of Gini mean values in the sense of harmonic mean," Acta Mathematica Scientia B, vol. 31, no. 3, pp. 1103-1112, 2011.

[12] W.-F. Xia, X.-H. Zhang, G.-D. Wang, and Y.-M. Chu, "Some properties for a class of symmetric functions with applications," Indian Journal of Pure and Applied Mathematics, vol. 43, no. 3, pp. 227-249, 2012.
[13] K. Guan, "Some properties of a class of symmetric functions," Journal of Mathematical Analysis and Applications, vol. 336, no. 1, pp. 70-80, 2007.

[14] W.-F. Xia and Y.-M. Chu, "Schur convexity and Schur multiplicative convexity for a class of symmetric functions with applications," Ukrainian Mathematical Journal, vol. 61, no. 10, pp. 1541-1555, 2009.

[15] W. Xia, G. Wang, and Y. Chu, "Schur convexity and inequalities for a class of symmetric functions," International Journal of Pure and Applied Mathematics, vol. 58, no. 4, pp. 435-452, 2010.

[16] N. N. Chan, "Schur-convexity for A-optimal designs," Journal of Mathematical Analysis and Applications, vol. 122, no. 1, pp. 1-6, 1987.

[17] W.-D. Jiang, "Some properties of dual form of the Hamy's symmetric function," Journal of Mathematical Inequalities, vol. 1, no. 1, pp. 117-125, 2007.

[18] F. Qi, J. Sándor, S. S. Dragomir, and A. Sofo, "Notes on the Schurconvexity of the extended mean values," Taiwanese Journal of Mathematics, vol. 9, no. 3, pp. 411-420, 2005.

[19] S. Wu, "Generalization and sharpness of the power means inequality and their applications," Journal of Mathematical Analysis and Applications, vol. 312, no. 2, pp. 637-652, 2005.

[20] A. W. Marshall, I. Olkin, and B. C. Arnold, Inequalities: Theory of Majorization and Its Applications, Academic press, New York, NY, USA, 2nd edition, 2011.

[21] X.-M. Zhang, "Schur-convex functions and isoperimetric inequalities," Proceedings of the American Mathematical Society, vol. 126, no. 2, pp. 461-470, 1998.

[22] C. Stepniak, "Stochastic ordering and Schur-convex functions in comparison of linear experiments," Metrika. International Journal for Theoretical and Applied Statistics, vol. 36, no. 1, pp. 291-298, 1989.

[23] F. K. Hwang and U. G. Rothblum, "Partition-optimization with Schur convex sum objective functions," SIAM Journal on Discrete Mathematics, vol. 18, no. 3, pp. 512-524, 2004/05.

[24] G. M. Constantine, "Schur convex functions on the spectra of graphs," Discrete Mathematics, vol. 45, no. 2-3, pp. 181-188, 1983.

[25] M. Merkle, "Convexity, Schur-convexity and bounds for the gamma function involving the digamma function," Rocky Mountain Journal of Mathematics, vol. 28, no. 3, pp. 1053-1066, 1998.

[26] A. Forcina and A. Giovagnoli, "Homogeneity indices and Schur-convex functions," Statistica, vol. 42, no. 4, pp. 529-542, 1982.

[27] M. Shaked, J. G. Shanthikumar, and Y. L. Tong, "Parametric Schur convexity and arrangement monotonicity properties of partial sums," Journal of Multivariate Analysis, vol. 53, no. 2, pp. 293-310, 1995

[28] J. E. Pecaric', F. Proschan, and Y. L. Tong, Convex Functions, Partial Orderings, and Statistical Applications, Academic Press, New York, NY, USA, 1992

[29] K. Guan, "A class of symmetric functions for multiplicatively convex function," Mathematical Inequalities \& Applications, vol. 10, no. 4, pp. 745-753, 2007.

[30] Y. Chu, X. Zhang, and G. Wang, "The Schur geometrical convexity of the extended mean values," Journal of Convex Analysis, vol. 15, no. 4, pp. 707-718, 2008.

[31] Y. Chu and Y. Lv, "The Schur Harmonic Convexity of the Hamy Symmetric Function and Its Applications," Journal of Inequalities and Applications, vol. 2009, Article ID 838529, 10 pages, 2009. 
[32] P. S. Bullen, Handbook of Means and their Inequalities, Kluwer Academic Publishers, Dordrecht, Netherlands, 2003.

[33] D. S. Mitrinovic', J. E. Pecaric', and V. Volenec, Recent Advances in Geometric Inequalities, Kluwer Academic Publishers Group, Dordrecht, Netherlands, 1989. 


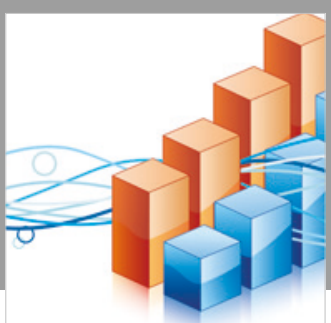

Advances in

Operations Research

\section{-n-m}
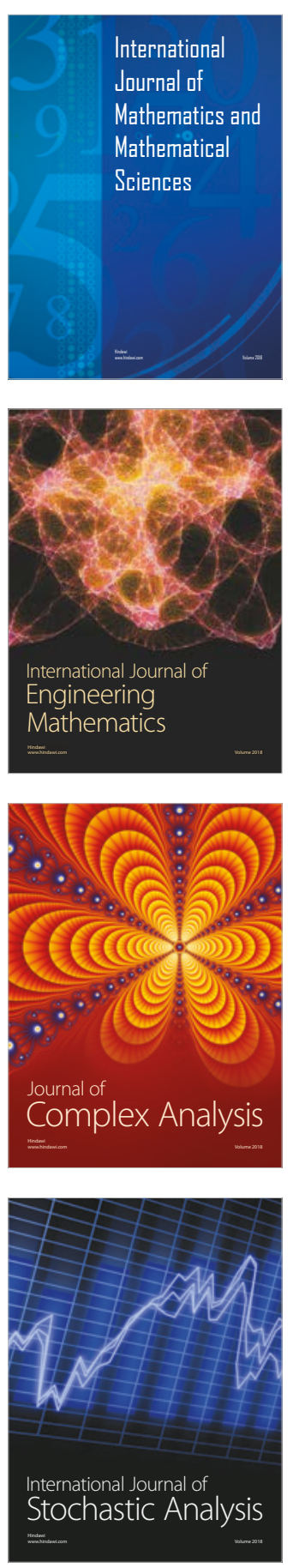
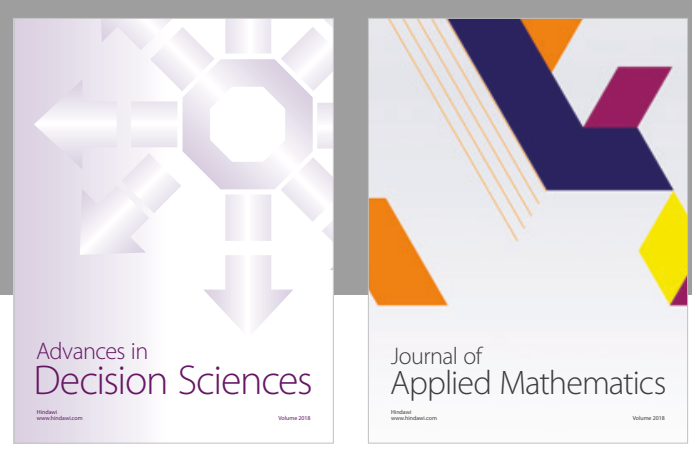

Journal of

Applied Mathematics
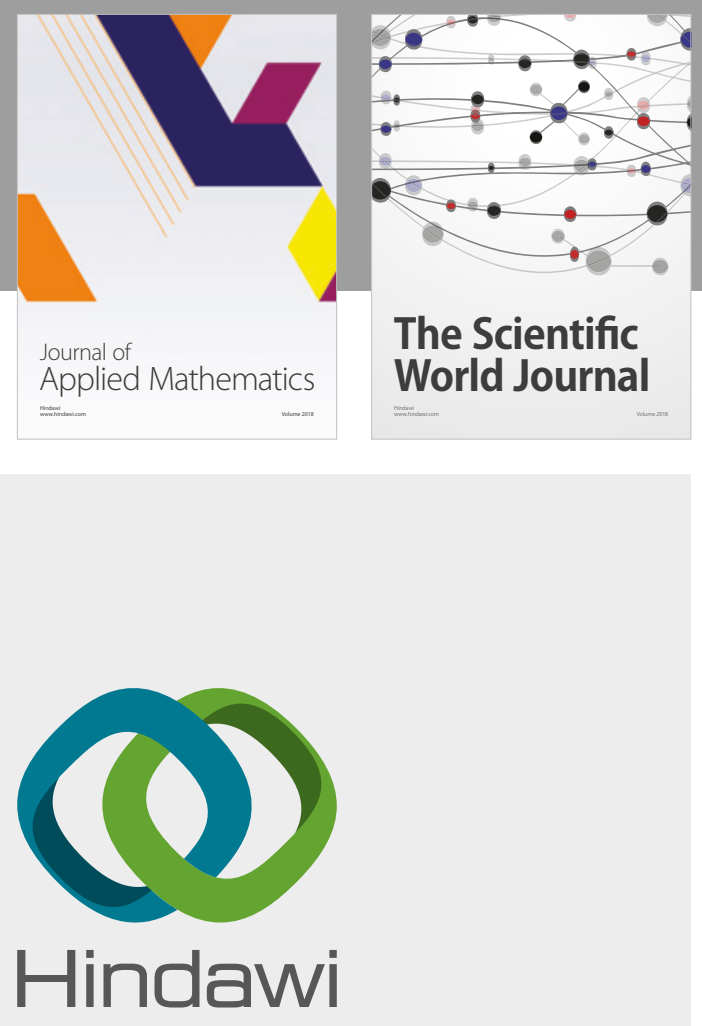

Submit your manuscripts at

www.hindawi.com

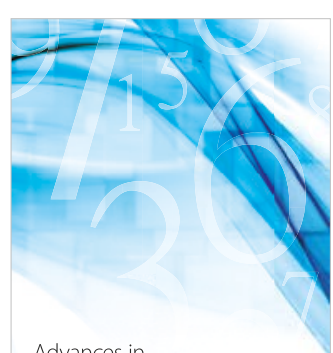

Advances in
Numerical Analysis
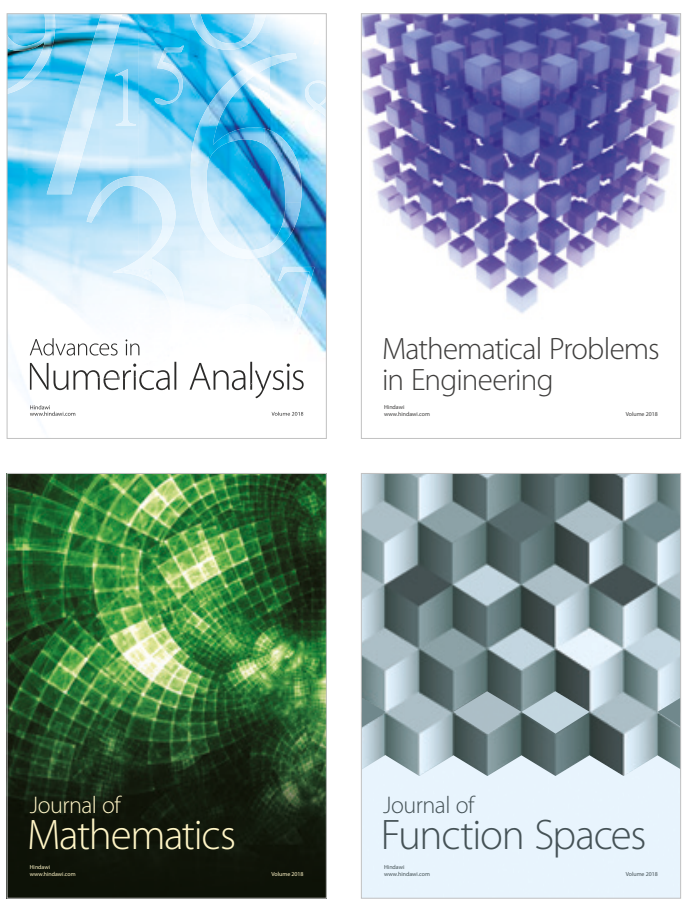

Mathematical Problems in Engineering

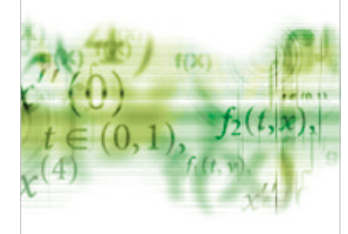

International Journal of

Differential Equations

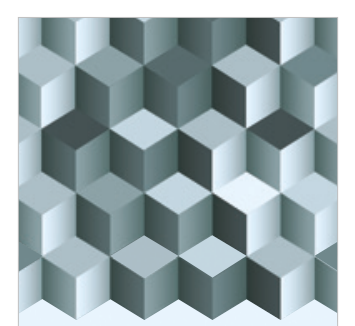

Journal of

Function Spaces

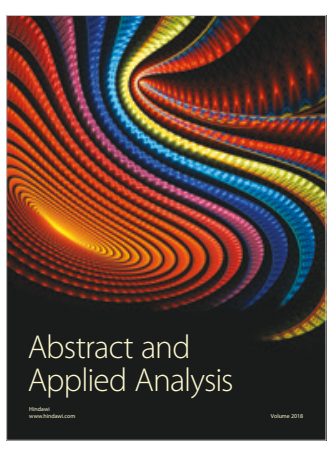

The Scientific

World Journal

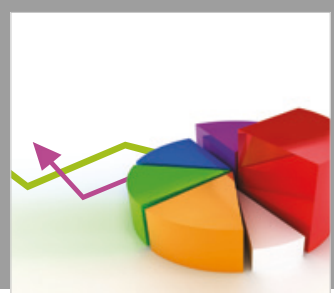

Journal of

Probability and Statistics
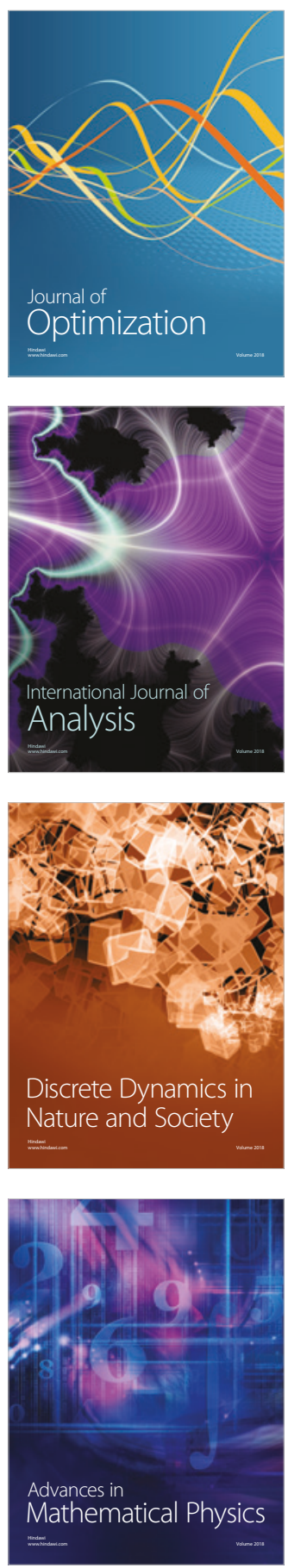